\title{
Síndrome de edema de la médula ósea de cadera
}

\author{
Marco Carrión $\mathrm{M}^{1}$, Pedro Pablo Amenábar $\mathrm{E}^{1}$, \\ Patricio Rodríguez $\mathrm{M}^{1}$, Ó scar Contreras $0^{2}$, Jaime Paulos $\mathrm{A}^{1}$.
}

\author{
Bone marrow edema \\ syndrome of the hip
}

Background: Bone marrow edema syndrome (BMES) is a rare clinical condition. Its etiology is unknown and it can be seen in different locations. In the case of the hip, avascular necrosis is the main differential diagnosis. Aim: To present our experience of BMES of the hip and a review of the literature. Patients and methods: Retrospective analysis of clinical records of patients with hip pain that met clinical, radiological, and magnetic resonance imaging criteria for BMES. Clinical outcome and clinical and radiological follow up are presented. Results: Two men and two women (one of them pregnant) aged 42, 48, 36 and 26 years old, fulfilled criteria. Treatment included limited weight bearing, non steroidal antiinflammatory drugs, intranasal calcitonin and physical therapy. Complete remission of symptoms was observed within five to seven months. At an average follow up of 36 months, all patients presented complete function of the hip, returning to their previous activity levels, with no new episodes of BMES. Conclusions: It is important to be aware of this condition as part of the differential diagnosis of hip pain to avoid aggressive and unnecessary diagnostic and therapeutic procedures (Rev Méd Chile 2004; 132: 947-54).

(Key Words: Bone marrow edema syndrome; Hip; Osteoporosis)

Recibido el 1 de marzo, 2004. Aceptado el 20 de mayo, 2004.

${ }_{1}^{1}$ Departamento de Ortopedia y Traumatología, Pontificia Universidad Católica de Chile.

${ }^{2}$ Departamento de Radiología, Pontificia Universidad Católica de Chile.

$\mathrm{E}$ síndrome de edema de la médula ósea (SEMO), que incluye la osteoporosis transitoria y la osteoporosis regional migratoria, es un cuadro clínico poco frecuente que afecta a personas de mediana edad, sin relación con enfermedades preexistentes y que tiene un curso benigno y autolimitado.

Correspondencia a: Dr. Pedro Pablo Amenábar E. Departamento de Ortopedia y Traumatología, Pontificia Universidad Católica de Chile. Lira 41, $2^{\circ}$ piso, Santiago, Chile. Teléfono: 56 (2) 3546846. Fax: 56 (2) 3546847. E mail: amenabar@med.puc.cl
Se caracteriza por dolor articular inespecífico, sin un evento desencadenante, pérdida focal más o menos pronunciada de la densidad mineral ósea en las radiografías, hipercaptación en la cintigrafía ósea, patrón de edema de la médula ósea en la resonancia magnética (RM) sin signos específicos de otra patología, y resolución clínica gradual y espontánea ${ }^{1-3}$.

En el caso de la cadera, el diagnóstico diferencial incluye necrosis avascular (NAV), fractura por estrés del cuello femoral, neoplasias, infecciones osteoarticulares (osteomielitis, artritis, tuberculosis), artritis inflamatorias, condromatosis sinovial o 
sinovitis villonodular ${ }^{4-9}$, con las posibles implicaciones que esto trae en cuanto a procedimientos diagnósticos y terapéuticos.

El objetivo de este trabajo es presentar nuestra casuística de SEMO de cadera y hacer una revisión de la literatura, con énfasis en las manifestaciones clínicas y el estudio de imágenes.

\section{Material y MÉTOdO}

Se identificaron los pacientes atendidos en nuestra institución entre los años 1997 y 2002, que cumplieron con los siguientes criterios: 1) Coxalgia sin relación a traumatismo, infección u otra causa demostrable. 2) Radiografía normal o con osteopenia localizada, sin compromiso del espacio articular y sin signos de otra patología. 3) Patrón de edema de la médula ósea en la resonancia magnética nuclear (RM) con disminución de la señal en T1 y aumento en T2 y STIR, sin signos sugerentes de NAV. 4) Resolución autolimitada del cuadro. 5) Seguimiento de por lo menos un año después de la desaparición del dolor sin otro diagnóstico que explique el cuadro.

Se revisó la historia clínica de cada paciente, tomando en cuenta las características demográficas y la sintomatología. Se registró el tiempo desde el inicio del cuadro hasta el diagnóstico definitivo y los tratamientos previos. Así mismo, se analizó los estudios de imágenes, que incluyeron radiografía convencional, cintigrafía ósea y $\mathrm{RM}$.

Se presenta la evolución del cuadro clínico, el tratamiento realizado y el resultado de los exámenes de imágenes de control. Se determinó si hubo aparición de cambios degenerativos, así como recurrencias en la misma u otra articulación. La capacidad funcional se determinó mediante la escala de cadera de Harris.

\section{RESULTADOs}

Se identificaron cuatro pacientes que cumplieron con los criterios clínicos y de imágenes: dos hombres y dos mujeres, una de ellas embarazada. La edad promedio fue 38 años (Tabla 1). Ninguno de ellos había tenido patologías previas en la cadera afectada.

Todos debutaron con dolor agudo, progresivo, que aumentaba con la actividad pero persistía incluso en reposo, sin respuesta a los antiinflamatorios no esteroidales ni a la fisioterapia. En dos casos el dolor se localizó en el trocánter mayor, en uno en la región inguinal y en otro en la región glútea.

El diagnóstico inicial fue necrosis avascular de la cabeza femoral en uno de los hombres y en la mujer no embarazada, a quienes se les había indicado un reemplazo total de cadera y acudieron a nuestra institución para una segunda opinión. En la mujer embarazada con dolor glúteo, el primer diagnóstico fue una lumbalgia; después del parto se identificó que el dolor provenía de la cadera izquierda y se realizó la evaluación de imágenes que llevó al diagnóstico definitivo. En el otro hombre se postuló una coxalgia en estudio, hasta que se diagnosticó el SEMO mediante RM.

El tiempo de latencia entre el inicio de los síntomas y el diagnóstico definitivo de SEMO fue en promedio 2,6 meses (Tabla 1).

Tabla 1. Características de cuatro pacientes con SEM O de cadera

\begin{tabular}{|cccccc|}
\hline & Edad y sexo & Lado & $\begin{array}{c}\text { Tiempo hasta el } \\
\text { diagnóstico* }\end{array}$ & Duración total* & Seguimiento* \\
\hline 1 & $36 \mathrm{~F}^{* *}$ & Izq & 1,5 & 5 & 74 \\
2 & $42 \mathrm{M}$ & Der & 2 & 6 & 29 \\
3 & $48 \mathrm{M}$ & Der & 2 & 7 & 24 \\
4 & $26 \mathrm{~F}$ & Izq & 5 & 6 & 17 \\
\hline
\end{tabular}

* En meses.

** En el octavo mes de su tercer embarazo. 
$\mathrm{Al}$ examen físico, todos los pacientes tuvieron limitación leve de la movilidad en los extremos del rango articular de la cadera afectada por dolor.

Las radiografías de pelvis mostraron osteopenia de la cabeza femoral en tres pacientes (Figura 1). En el otro, las radiografías tomadas a las dos y ocho semanas, se consideraron normales, por lo que se realizó una cintigrafía ósea a las ocho semanas que mostró un aumento difuso de la captación en la cabeza, cuello y región intertrocantérica. En los cuatro casos, el diagnóstico definitivo se realizó con resonancia magnética, que demostró el patrón característico de edema difuso de la médula ósea (Figura 2).

Una vez realizado el diagnóstico, se prescribió descarga de la extremidad afectada y antiinflamatorios no esteroidales. En dos pacientes se indicó además el uso de calcitonina intranasal, que alivió parcialmente las molestias. El tiempo de duración total de la sintomatología varió entre cinco y siete meses (promedio 6 meses) (Tabla 1), luego de lo cual los pacientes retornaron a sus actividades de la vida diaria sin ninguna secuela.

En un seguimiento promedio de 36 meses (rango, 17-74 meses), todas las caderas fueron clínicamente normales (100 puntos en la escala de Harris). No hubo recurrencias de SEMO de cadera u otras localizaciones ni otra patología de cadera ipsilateral o contralateral.
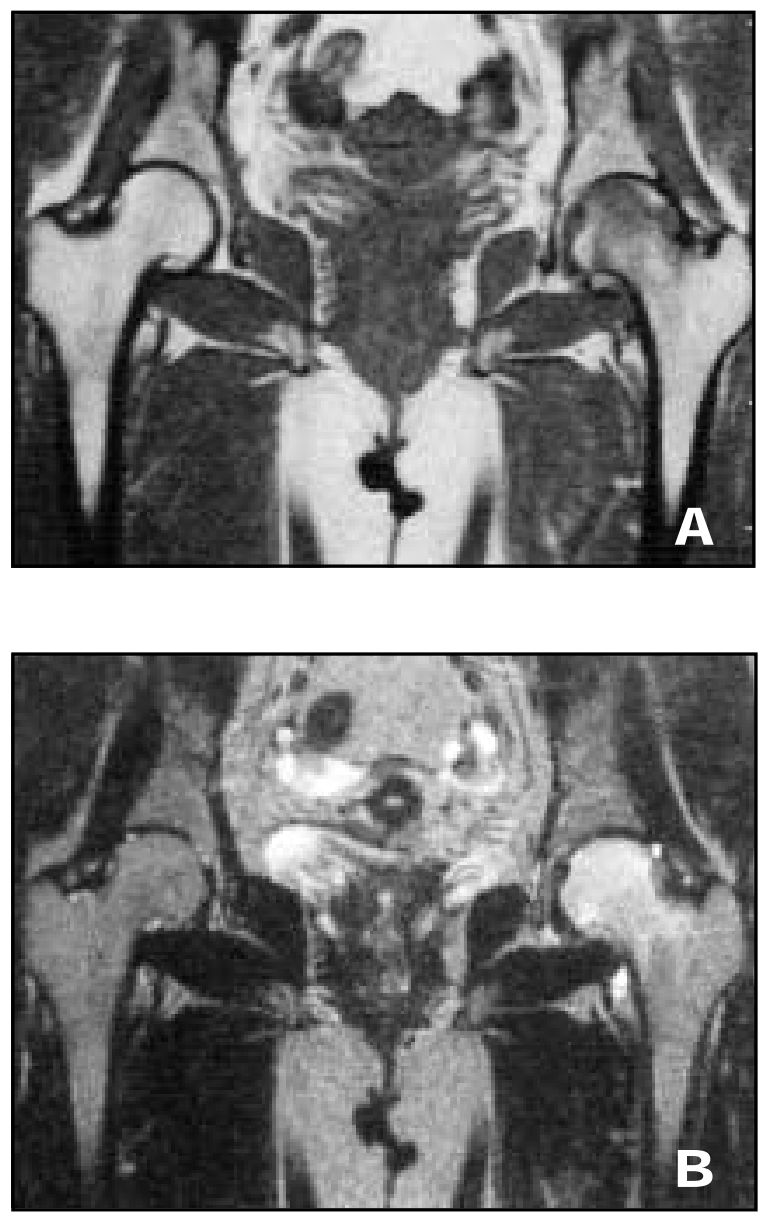

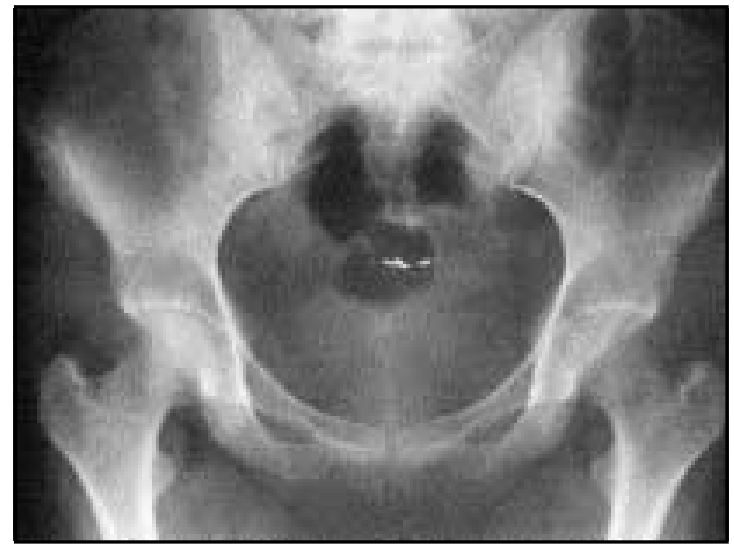

Figura 1. Radiografía de pelvis ántero-posterior que evidencia acentuada osteopenia de la cabeza femoral izquierda que se extiende hasta la región intertrocantérica (cabeza fantasma).

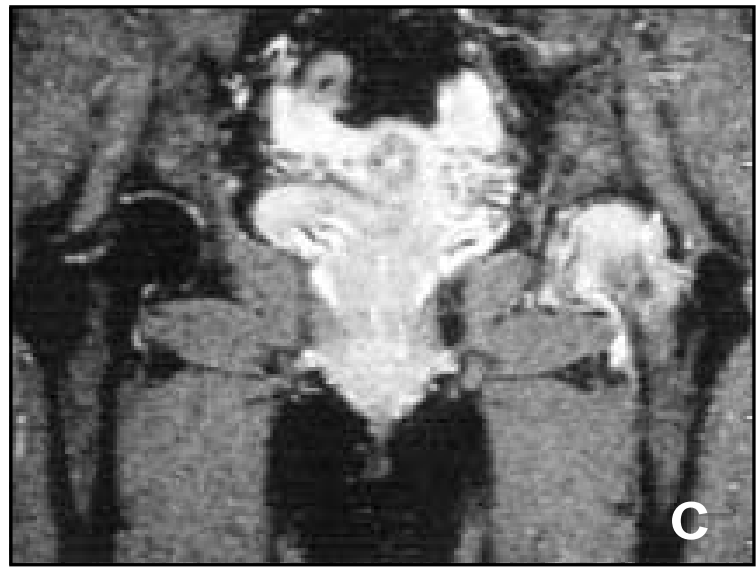

Figura 2. Imágenes de RM que muestran disminución de la señal focal en cabeza y cuello femoral izquierdos en T1 (a) y aumento en T2 (b) y STIR (c). 
En tres casos se realizó RM de control y se observó la normalización de las imágenes en la cadera afectada (Figura 3). En el cuarto paciente se realizó una cintigrafía ósea posterior a la resolución del cuadro, la cual fue completamente normal. Al último seguimiento todos los pacientes presentaron radiografías de cadera normales.

\section{DisCUSIÓN}

En 1947, Ravault et al describieron por primera vez un cuadro de estas características en la extremidad superior ${ }^{10}$. En 1959, Curtiss y Kincaid reportaron tres casos de desmineralización transitoria de la cadera asociada con el tercer trimestre del embarazo ${ }^{11} \mathrm{y}$, en 1968, Lequesne lo denominó «osteoporosis transitoria» de la cadera ${ }^{12}$.

A través de los años ha recibido diversos nombres, entre ellos: algodistrofia de cadera ${ }^{13,14}$, desmineralización transitoria ${ }^{11}$, osteoporosis regio-
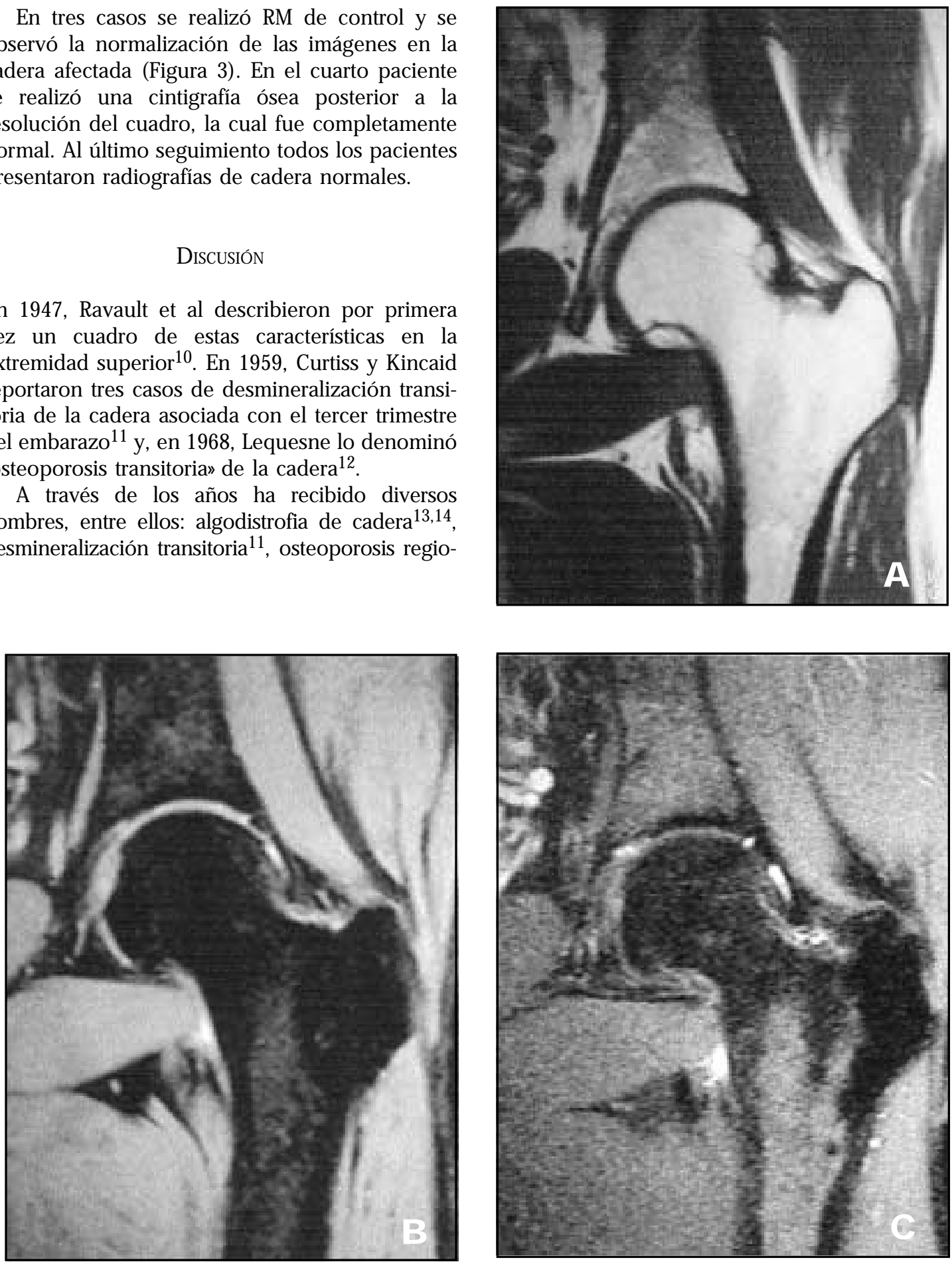

Figura 3. Imágenes de RM de control del mismo paciente de la Figura 2, dos meses después de la resolución del cuadro, con normalización de la señal en T1 (a), T2 (b) y STIR (c). 
nal transitoria ${ }^{5}$, osteoporosis regional migratoria ${ }^{15}$, osteoporosis regional idiopática ${ }^{16}$, osteoporosis transitoria dolorosa ${ }^{17}$, osteolisis migratoria ${ }^{18,19}$, osteopenia transitoria ${ }^{20}$, síndrome de edema de la médula ósea1,2,21-24.

Se ha propuesto que existen al menos dos formas clínicas de este síndrome, que se han conocido tradicionalmente como osteoporosis transitoria y osteoporosis regional migratoria ${ }^{4,7,25-30}$. La osteoporosis transitoria es una forma localizada que afecta principalmente la cadera (70\% de casos), seguida en frecuencia por la rodilla, tobillo y pie y otras articulaciones ${ }^{5}$; con frecuencia afecta a mujeres en el tercer trimestre del embarazo y puede ser bilateral o con recurrencias en la misma articulación ${ }^{30,31}$. La osteoporosis regional migratoria, descrita por Duncan et al en $1967^{18}$, presenta tanto recurrencias como migración y prefiere hombres de mediana edad. Compromete predominantemente tobillo y pie y, siguiendo en frecuencia, rodilla, cadera, columna y extremidades superiores ${ }^{5,17}$; los síntomas son más severos y de mayor duración, y hay osteopenia radiológica más precoz, difusa y masiva $^{29,30}$.

La etiología del SEMO es desconocida. Se han postulado diversas hipótesis, entre ellas: una forma frustra de distrofia simpática refleja ${ }^{4,12,14,25,32}$, compresión del nervio obturador por la cabeza del feto $^{11}$, denervación local ${ }^{5,15}$, obstrucción del retorno venoso ${ }^{1,26,33,34}$, fenómenos isquémicos transitorios que pudieran tener relación con tabaquismo, anticuerpos antifosfolípidos o dislipidemias ${ }^{9,35}$, microtrauma con un fenómeno acelerador regional» $3,30,36$, osteopenia por desuso ${ }^{9}$, un estímulo desconocido de los osteoclastos, que pudiera ser viral $^{35}$, cambios en el metabolismo del calcio ${ }^{8,37}$, factores hormonales ${ }^{38}$, etc.

La teonía más discutida es que se trate de una forma inicial y reversible de $\mathrm{NAV}^{1,24,30,34,39}$, aunque hay diversas observaciones imagenológicas e histológicas que se contraponen a esta hipótesis $22,35,40,41$.

Hasta ahora, el único factor predisponente conocido es el embarazo y la incidencia parece ser mayor en pacientes con osteogénesis imperfecta que en la población general ${ }^{42,43}$. Algunos autores han reportado casos en los que se asoció con osteoporosis generalizada ${ }^{3,30}$ pero otros han encontrado una densidad mineral ósea normal ${ }^{2,34}$.

El SEMO de cadera afecta predominantemente a personas de sexo masculino (proporción 3:1) de entre 30 y 60 años de edad. En las mujeres, ocurre casi exclusivamente en el tercer trimestre del embarazo o en el puerperio y es más común en primigestas $^{5,9,29,31,35,44}$. Se ha comunicado otra variante que puede afectar a niños o mujeres en el primer trimestre del embarazo, que tiende a ser bilateral y puede tener radiografías normales $^{20,29,45}$.

Se manifiesta clínicamente por dolor progresivo, incapacitante, exacerbado con la marcha pero presente incluso en reposo 29,35 . La marcha puede ser claudicante, antiálgica o imposible sin apoyo externo. Hay restricción leve del rango articular por dolor. Un 30\% de los pacientes puede tener síntomas en la cadera contralateral ${ }^{4}$. Destaca la discrepancia entre la marcada incapacidad funcional y los escasos hallazgos clínicos ${ }^{30}$. Se han reportado fracturas tanto espontáneas como iatrogénicas en hombres y mujeres ${ }^{11,35,37,38,46-48}$. La sintomatología suele durar entre tres y diez meses, con un promedio de siete meses $4,5,9,27,29,30,35$, aunque se ha descrito una duración de hasta 45 meses $^{5}$.

Los cambios radiográficos aparecen entre tres y seis semanas del inicio del dolor 3,4,12,29,30. Hay una radiolucencia difusa uniforme desde la cabeza femoral hasta la región intertrocantérica, que puede verse incluso como «cabeza fantasma»35. No hay disminución del espacio articular, colapso subcondral o imágenes quísticas ${ }^{29}$ (Figura 1). La densidad ósea se recupera poco después de la mejoría clínica, aunque las trabéculas pueden quedar engrosadas e irregulares indefinidamente ${ }^{5}$.

La cintigrafía ósea con $\mathrm{Tc}^{99}$ fue importante para el diagnóstico precoz antes del advenimiento de la RM. Se observa un aumento difuso y homogéneo de la captación en la cabeza y cuello femoral $29,35,49$. Es sensible pero poco específica.

La RM permite un diagnóstico precoz, incluso a las $48 \mathrm{~h}$ del inicio del dolor ${ }^{50}$, detecta casos sin osteopenia radiográfica y excluye otros diagnósticos. El patrón de edema de la médula ósea consiste en disminución uniforme y difusa de la señal en T1 y aumento de la señal en T2 y STIR en la cabeza y cuello que se extiende a la región intertrocantérica (Figura 2) 6,7,29,35,50-54. Es común encontrar derrame articular. Hallazgos menos frecuentes son un patrón de edema moteado ${ }^{29}$ y una línea subcondral hipointensa en T1 que podría corresponder a una pequeña fractura por insuficiencia ${ }^{40,55}$. 
El edema por sí solo no es específico y se puede ver en NAV, contusión ósea, infección o neoplasia infiltrante ${ }^{1,56}$, pero el curso clínico de estos cuadros es diferente. Además, en la NAV precoz, el edema está focalizado en la cabeza formando una banda alrededor de una zona hipointensa de hueso muerto en T2 (signo del doble anillo) ${ }^{35}$. El edema difuso que llega a la región intertrocantérica no se observa en etapas tempranas pero puede verse cuando se produce la fractura subcondral ${ }^{41}$, la cual es claramente visible en la RM.

Los exámenes de laboratorio suelen ser normales, aunque se han descritos casos de aumento de la velocidad de sedimentación globular ${ }^{5,11,29}$ y de los niveles de fosfatasa alcalina sérica ${ }^{4}$ e hidroxiprolina urinaria ${ }^{57}$.

Las biopsias óseas muestran trabéculas adelgazadas, laxamente distribuidas, recubiertas de osteoide y osteoblastos activos. Hay lagunas vacías, pero también hay osteoblastos viables en otras, a diferencia de la NAV. En los espacios medulares se observa líquido exudativo, congestión y hemorragia intersticial, fragmentación de los adipocitos, con áreas de proliferación de vasos sanguíneos, infiltrado inflamatorio crónico y fibrosis leve $e^{1,7,34,40}$. No

\section{REFERENCIAS}

1. Pienk H, Hofmann S, Eschberger J, Gstettner M, KRAMER J, SchNeIDER W ET AL. Histomorphology and bone morphometry of the bone marrow edema syndrome of the hip. Clin Orthop 1997; 334: 73-84.

2. Calvo E, Fernández-Yruegas D, Alvarez L Core decompression shortens the duration of pain in bone marrow oedema syndrome. Int Orthop 2000; 24: 88-91.

3. Trevisan C, ORtolani S. Bone loss and recovery in regional migratory osteoporosis. Osteoporosis Int 2002; 13: 901-6.

4. Kaplan SS, Stegman CJ. Transient osteoporosis of the hip. Case report and review of the literature. J Bone Joint Surg Am 1985; 67: 490-3.

5. Lakhanpal S, Ginsburg WW, Luthra HS, Hunder GG. Transient regional osteoporosis. A study of hay osteoporosis ni osteolisis histológica, sino desmineralización y edema medular ${ }^{1}$.

Se han publicado diferentes opciones de tratamiento ${ }^{4}$. En general se utilizan medidas sintomáticas, como reposo en cama, marcha con descarga, tracción, fisioterapia y antiinflamatorios no esteroidales $5,9,27,35$. Varios autores han reportado el uso de corticoides $5,12,18,28,32$, calcitonina ${ }^{28-30,32}$, bifosfonatos $^{30} 0$ iloprost $^{23}$. En casos rebeldes se ha utilizado bloqueos simpáticos ${ }^{12,32}$. No se ha demostrado que ninguno de estos tratamientos afecte el curso de la enfermedad.

Se ha descrito una mejoría significativa del dolor con perforaciones de la cabeza femoral ${ }^{34}$ y una disminución de la duración de la sintomatología desde 7,4 a 3,7 meses en una serie ${ }^{2}$ y desde 6 a 2 meses en otra ${ }^{58}$. Pero se trata de un tratamiento agresivo para un cuadro autolimitado ${ }^{35}$ y además se han comunicado complicaciones iatrogénicas de la biopsia o de la descompresión 11,37,38,46.

En conclusión, el SEMO es un cuadro raro con escasas complicaciones, por lo que se aconseja el manejo sintomático del dolor y tranquilizar al paciente hasta la resolución de la sintomatología. El diagnóstico y el seguimiento deben ser cuidadosos para no pasar por alto otras patologías, especialmente la NAV de cadera.

56 cases and review of the literature. Ann Intern Med 1987; 106: 444-50.

6. Takatori $\mathrm{Y}$, Kokubo T, Ninomita S, Nakamura $T$, OKUTSU I, Kamogawa M. Transient osteoporosis of the hip. Magnetic resonance imaging. Clin Orthop 1991; 271: 190-4.

7. Potter $\mathrm{H}$, Morán $\mathrm{M}$, Schneider $\mathrm{R}$, Bansal $\mathrm{M}$, SHERMAN C, MARKISZ J. Magnetic resonance imaging in diagnosis of transient osteoporosis of the hip. Clin Orthop 1992; 280: 223-9.

8. Bijl M, Van Leeuwen MA, Van RJjswijk MH. Transient osteoporosis of the hip: Presentation of (a) typical cases and a review of the literature. Clin Exp Rheumatol 1999; 17: 601-4.

9. Crespo E, Sala D, Crespo R, Silvestre A. Transient osteoporosis. Acta Orthop Belga 2001; 67: 330-7.

10. Ravault PP, Gainet P, Perther L, Emery J, Carrer F. Rhumatismes chroniques de la main et rhumatis- 
me neurotrophique du membre superieur. J Med Lyon 1947; 28: 363.

11. CuRTISS PH JR, KINCAID WE. Transitory demineralization of the hip in pregnancy. J Bone Joint Surg Am 1959; 41: 1327-33.

12. LeQUeSNe M. Transient osteoporosis of the hip: A nontraumatic variety of Sudeck's athrophy. Ann Rheum Dis 1968; 27: 463-71.

13. LeQuesne M. L'algodystrophie de la hanche. Presse Med 1968: 76: 973.

14. Doury P. Bone marrow oedema, transient osteoporosis, and algodistrophy. J Bone Joint Surg $\mathrm{Br}$ 1994; 76: 993.

15. McCord WC, Nes KM, Campion DS, Louie JS. Regional migratory osteoporosis: A denervation disease. Arthritis Rheum 1978; 21: 834-8.

16. NAIDES SJ, ResNicK D, ZvaIFLER NJ. Idiopathic regional osteoporosis: A clinical spectrum. J Rheumatol 1985; 12: 763-8.

17. Langloh ND, Hunder GG, Riggs BL, Kely PJ. Transient painful osteoporosis of the lower extremities. J Bone Joint Surg Am 1973; 55: 1188-96.

18. Duncan H, Frame B, Frost HM, Arnstein AR. Migratory osteolisis of the lower extremities. Ann Intern Med 1967; 66: 1165-73.

19. Strashun A, Chayes Z. Migratory osteolysis. J Nucl Med 1979; 20: 129-32.

20. Nicol RO, Wiшамs PF, HiL DJ. Transient osteopaenia of the hip in children. J Pediatr Orthop 1984; 4: 590-2.

21. Wilson AJ, Murphy WA, Hardy DC, Totty WG. Transient osteoporosis: Transient bone marrow edema? Radiology 1988; 167: 757-60.

22. Solomon L. Bone marrow oedema syndrome. J Bone Joint Surg Br 1993; 75: 175-6.

23. Aigner N, Petje G, Schneider W, Krasny C, Grill F, LANDSIEDL $F$. Juvenile bone marrow oedema of the acetabulum treated by iloprost. Case report. J Bone Joint Surg Br 2002; 84: 1050-2.

24. Koo KH, Ahn IO, Song HR, Kim SY, Jones JP. Increased perfusion of the femoral head in transient bone marrow edema syndrome. Clin Orthop 2002; 402: 171-5.

25. SWESEY RL. Transient osteoporosis of the hip, foot and knee. Arthritis Rheum 1970; 13: 858-68.

26. RoSen RA. Transitory demineralization of the femoral head. Radiology 1970; 94: 509-12.

27. SHIFRIN IZ, REIS ND, ZiNMAN H, Besser MI. Idiopathic transient osteoporosis of the hip. J Bone Joint Surg Br 1987; 69: 769-73.
28. Aguilera S, Cortés C, Martínez V. Osteoporosis regional migratoria. Efecto de tratamiento con calcitonina. Presentación de 2 casos. Rev Méd Chile 1994; 122: 1045-51.

29. Ribera Zabalbeascoa J, Santos Rodas A, Melia Sousa M, Uceda Carrascosa P, Benito Caparros M. Transient osteoporosis of the hip. Int Orthop 1999; 23: 244-6.

30. Trevisan C, Ortolani S, Montelone M, Marinoni EC. Regional migratory osteoporosis: A pathogenetic hypothesis based on three cases and a review of the literature. Clin Rheumatol 2002; 21: 418-25.

31. Valenzueia F, Aris H, Jacobe山 S. Transient osteoporosis of the hip. J Rheumatol 1977; 4: 59-64.

32. Cuchacovich M, Vial P, Trewhela R, Gatica H. Osteoporosis regional transitoria y distrofia simpática refleja. ¿Entidades distintas o variantes clínicas de una misma enfermedad? Rev Méd Chile 1995; 123: 485-92.

33. Bray ST, Partain CL, Teates CD, Guilford WB, WiLAmson BR, McLaughun RC. The value of the bone scan in idiopathic regional migratory osteoporosis. J Nucl Med 1979; 20: 1268-71.

34. Hofmann S, Engel A, Neuhold A, Leder K, Kramer J, PIENK H. Bone marrow oedema syndrome and transient osteoporosis of the hip. An MRI-controlled study of treatment by core decompression. J Bone Joint Surg Br 1993; 75: 210-6.

35. GueRRA JJ, SteinBERg ME. Distinguishing transient osteoporosis from avascular necrosis of the hip. J Bone Joint Surg Am 1995; 77: 616-24.

36. Beauleu JG, Razzano CD, Levine RB. Transient osteoporosis of the hip in pregnancy: Review of the literature and a case report. Clin Orthop 1976; 115: 165.

37. Brodell JD, Burns JE, Heipie KG. Transient osteoporosis of the hip of pregnancy. Two cases complicated by pathological fracture. J Bone Joint Surg Am 1989; 71: 1252-7.

38. FingeRoth RJ. Successful operative treatment of a displaced subcapital fracture of the hip in transient osteoporosis of pregnancy. A case report and review of the literature. J Bone Joint Surg Am 1995; 77: 127-31.

39. Turner DA, Templeton AC, Seizer PM, Rosenberg AG, PETASNICK JP. Femoral capital osteonecrosis: MR finding of diffuse marrow abnormalities without focal lesions. Radiology 1989; 171: 135-40.

40. Yамамото $T$, Kubo T, Hirasawa $Y$, Noguchi $Y$, Iwamoto Y, Sueishi K. A clinicopathologic study of 
transient osteoporosis of the hip. Skeletal Radiol 1999; 28: 621-7.

41. Kim YM, Oh HC, Kim HJ. The pattern of bone marrow oedema on MRI in osteonecrosis of the femoral head. J Bone Joint Surg Br 2000; 82: 83741.

42. Noorda RJP, Van Der AA JPW, Wuisman PIJ, David EFL, Lips PTA, VAN DeR ValK P. Transient osteoporosis and osteogenesis imperfecta. A case report. Clin Orthop 1997; 337: 249-55.

43. Karagrevis CB, Ainscow DAP. Transient osteoporosis of the hip associated with osteogenesis imperfecta. J Bone Joint Surg Br 1998; 80: 54-5.

44. Pantazo poulos T, Exarchou E, Hartofilakidis-GaroFALDIS G. Idiopathic transient osteoporosis of the hip. J Bone Joint Surg Am 1973; 55: 315-21.

45. Nishiyama K, SAKAMAKI T. Transient osteopenia of the hip in children. Clin Orthop 1992; 275: 199203.

46. MoRÁn MC. Iatrogenic femoral neck fracture in transient osteoporosis of the hip. A case report. Clin Orhtop 1995; 316: 207-10.

47. Junk S, Ostrowski M, KoKoszczynski L. Transient osteoporosis of the hip in pregnancy complicated by femoral neck fracture. A case report. Acta Orhtop Scand 1996; 67: 69-70.

48. FoKTER SK, Vengust V. Displaced subcapital fracture of the hip in transient osteoporosis of pregnancy. Int Orthop 1997; 21: 201-3.

49. O'Mara RE, Pinals RS. Bone scanning in regional migratory osteoporosis. Radiology 1970; 97: 579-81.
50. Daniel WW, Sanders PC, Alarcón GS. The early diagnosis of transient osteoporosis by magnetic resonance imaging. J Bone Joint Surg Am 1992; 74: 1262-4.

51. Alarcón GS, Sanders C, Daniel WW. Transient osteoporosis of the hip: Magnetic resonance imaging. J Rheumatol 1987; 14: 1184-9.

52. BLoem JL. Transient osteoporosis of the hip. MR imaging. Radiology 1988; 167: 753-5.

53. Wilson AJ, Murphy WA, HaRdy DC, TotTy WG. Transient osteoporosis: Transient bone marrow edema? Radiology 1988; 167: 757-60.

54. Urbanski SR, Lange EE, EscheNRoeder HC. Magnetic resonance imaging of transient osteoporosis of the hip. A case report. J Bone Joint Surg Am 1991; 73: 451-5.

55. Miyanishi K, Yamamoto T, Nakashima $\mathrm{Y}$, Shuto T, Jingushi S, Noguchi Y et aL. Subcondral changes in transient osteoporosis of the hip. Skeletal Radiol 2001; 30: 255-61.

56. Kubo T, Yamamoto T, Inoue $\mathrm{S}$, Hori M, Ueshima $\mathrm{K}$, IwAмото Y ET AL. Histological findings of bone marrow edema pattern on MRI in osteonecrosis of the femoral head. J Orthop Sci 2000; 5: 520-3.

57. Bramlett KW, Kiman JT, Nasca RJ, Danel WW. Transient osteoporosis. Clin Orthop 1987; 222: 197-202.

58. Hofmann S, Schneider W, Breitenseher M, URban M, PienK H. Die «ransiente osteoporose» als reversible sondeform der hüftkopfnekrose. Orthopäde 2000; 29: 411-9. 